\title{
VLSI Circuits for Adaptive Digital Beamforming in Ultrasound Imaging
}

\author{
Mustafa Karaman, Abdullah Atalar, and Hayrettin Köymen
}

\begin{abstract}
For phased-array ultrasound imaging, alternative beamforming techniques and their VLSI circuits are studied to form a fully digital receive front-end hardware. In order to increase the timing accuracy in beamforming, a computationally efficient interpolation scheme to increase the sampling rate is examined. For adaptive beamforming, a phase aberration correction method with very low computational complexity is described. Image quality performance of the method is examined by processing the non-aberrated and aberrated phased-array experimental data sets of an ultrasound resolution phantom. A digital beamforming scheme based on receive focusing at the raster focal points is examined. The sector images of the resolution phantom, reconstructed from the phased-array experimental data by beamforming at the radial and raster focal points, are presented for comparison of the image resolution performances of the two beamforming schemes. VLSI circuits and their implementations for the proposed techniques are presented.
\end{abstract}

\section{INTRODUCTION}

$\mathbf{P}$ HASED-ARRAY ultrasound imaging techniques have been extensively used in modern medicine for diagnostic purposes. In reconstruction of phased-array ultrasound images, short bursts of ultrasound are transmitted, and echoes reflected from internal structures of body are received by a phased-array transducer. The imaging plane is scanned by beamforming - electronically steering and focusing the array-both in transmit and receive modes. In transmit mode, the beamforming process is performed at every scan angle, while in receive mode, it is dynamically repeated for every image point [1]. Design of the transmit beamforming circuitry is relatively easy, since each transducer can be fired by digital timing, while design of the receive beamforming circuitry is an involved task and has been the subject of considerable research [2], [3].

Real-time phased-array ultrasound beamforming involves a significant amount of electronic signal processing at video rates. Receive beamforming hardware based on analog circuitry is bulky and expensive. Recent developments in digital integrated circuit technology motivates research on advanced digital beamforming techniques based on special purpose VLSI circuits. [3]-[6]. In the design of such circuits, it is important to examine the beamforming algorithm performance, which critically affects image resolution [7], and the feasibility

\footnotetext{
Manuscript received July 14, 1992; revised May 17, 1993. This work was partially supported by the Turkish Scientific and Research Council, TUBITAK. The associate editor responsible for coordinating the review of this paper and recommending its publication was Dr. R. W. Martin.

The authors are with the Department of Electrical and Electronics Engineering, Bilkent University, Ankara, 06533 Turkey.

IEEE Log Number 9213399.
}

of algorithm for VLSI implementation [8]. In this study, we consider three major problems in beamforming: delay quantization, phase aberration, and receive focusing used for image reconstruction. We propose new digital beamforming schemes, and present VLSI circuits for their realizations.

Delay quantization has a significant undesired effect on the transmit and receive responses of the system, which results in an increase in the side lobe levels of the array response [9]. Accuracy of timing information used in beamforming can be increased using fast analog-to-digital converters (ADC), with relatively high cost. It can be also solved by employing digital upsampling (increasing the sampling rate by interpolation) techniques [10]. Since the timing accuracy in beamforming is much more critical than the amplitude accuracy, a simple upsampling scheme can be used for this purpose. As a costeffective solution for high timing accuracy in beamforming, we examine a linear interpolation scheme with coefficients in discrete powers-of-two space [11].

Computation of the timing information required for the beamforming is based on the assumption that the image plane is composed of uniform soft tissue. This assumption, however, is not valid in general, and causes significant phase errors [12], and hence degradation in image resolution. The solution of this problem involves phase error correction using an adaptive beamforming technique. The algorithm used for this purpose must be computationally efficient for real-time applications. As a solution to this problem, we study a phase aberration correction technique with very low computational complexity, based on the time delay estimation via the minimization of sum of absolute differences between the samples of adjacent array elements.

In conventional ultrasound imaging, receive beamforming is carried out at the radial focal points. The radial data is converted to the raster data which correspond to the display pixels in rectangular coordinates. The conversion process, called scan conversion [13], imposes a significant hardware overhead and may degrade the image quality. In order to eliminate the scan conversion, we examine an alternative digital beamforming scheme based on receive focusing at the raster points.

In the next section, a digital front-end hardware architecture is outlined together with the description of the linear interpolation scheme. In Section III, the phase aberration correction method is presented. Section IV examines the beamforming at the raster focal points. In all cases, the hardware architectures are also discussed in detail. The VLSI implementations are presented in Section V. 


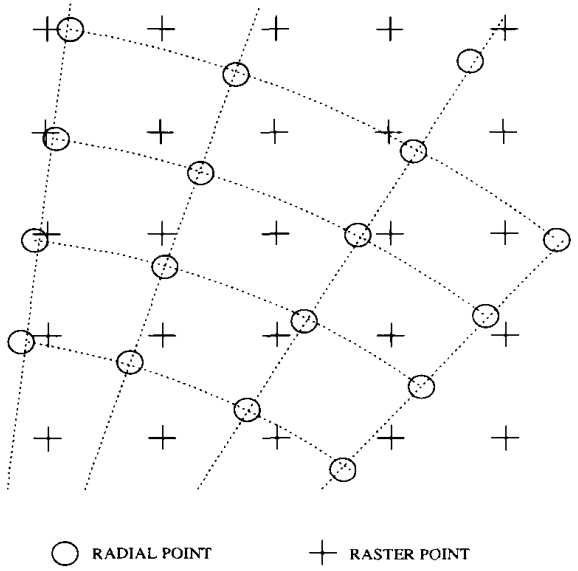

Fig. 1. Radial and raster focal points.

\section{Digital Front-End HaRdware}

In phased-array digital receive beamforming, radio frequency (RF) echo signals received by the phased-array elements are sampled using ADC's, and then all signal processing operations are handled by digital electronics. An architecture showing the processing units for the digital front end is depicted in Fig. 2. The timing accuracy in beamforming and in phase aberration correction is increased by upsampling. The phase aberration estimation is performed on the sampled data and then it is used to correct the beamforming timing computed for the receive focal points. The re-sampling process prior to beamforming is required to select the samples corresponding to the focal point. Finally, the samples corresponding to the focal point are synchronized and added to complete the beamforming. The signal value of the focal point is obtained at the output of the beamformer unit.

The sampling rate can be increased using baseband demodulation or bandpass interpolation techniques [10], [3]. The realization of the former technique requires mixing and lowpass filtering, while the latter requires only interpolation (zero padding and filtering). Since the level of time quantization is much more critical than the level of the amplitude quantization, a simple upsampling scheme such as a linear interpolation can be used for upsampling to increase the timing accuracy. In the linear interpolation, a new sample, point $C$, between two existing samples, points $A$ and $B$, can be interpolated as

$$
C=\frac{T_{1} A+\left(T_{2}-T_{1}\right) B}{T_{2}}
$$

where $T_{1}$ and $T_{2}$ are the time distances between $C$ and $B$, and $A$ and $B$, respectively. For hardware realization, this expression can be further simplified by expressing the coefficients $T_{1}$ and $T_{2}$ in the sum of powers-of-two form [11]. To do that we need to quantize $T_{1}$ and $T_{2}$. Choosing 8 quantization levels for $T_{2}$ is tolerable for both the beamforming and phase aberration correction purposes [14]. Thus, the coefficients $T_{1}$ and $T_{2}$ can be expressed as

$$
T_{1}=2^{p_{1}}+(-1)^{s_{1}} 2^{q_{1}} \quad T_{2}=2^{p_{2}}+(-1)^{s_{2}} 2^{q_{2}}
$$

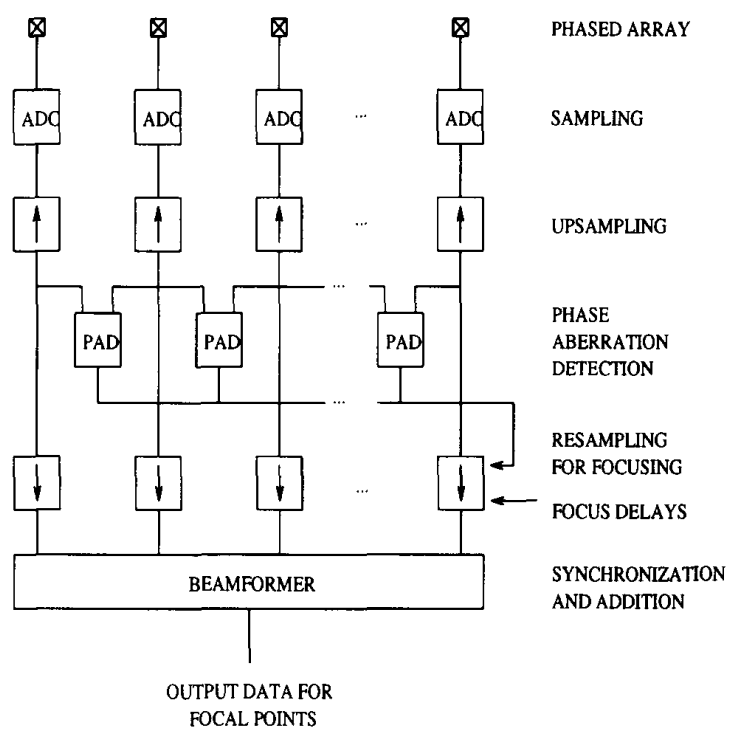

Fig. 2. Block diagram of the digital receive front-end hardware.

where $p_{1}, q_{1}, s_{1}, p_{2}, q_{2}$, and $s_{2}$ are non-negative integer numbers. As a result, the realization of the interpolation is reduced to three shift and three addition operations.

The hardware structure shown in Fig. 3 is developed for the linear interpolation scheme. It takes two input samples, $A$, and $B$, and the value of time distance to the point at which the interpolation will be performed. It generates the interpolated sample, $C$, as its output. The power coefficients for representation of $T_{1}$ and $T_{2}-T_{1}$, are generated by decoding $T_{1}$. The resultant signals, $p_{1}, q_{1}, s_{1}, p_{2}, q_{2}$, and $s_{2}$, are used as the control signals for shift-left operations on the input samples. Then, the appropriately shifted versions of each sample are added to realize the multiplications, $T_{1} A=$ $\left(2^{p_{1}}+(-1)^{s_{1}} 2^{q_{1}}\right) A$ and $\left(T_{2}-T_{1}\right) B=\left(2^{p_{2}}+(-1)^{s_{2}} 2^{q_{2}}\right) B$. Then, the interpolated sample is obtained by adding these two results and performing a shift right operation on the output. The interpolator scheme is designed in five pipelined levels to achieve a high speed.

\section{Phase Aberration Correction}

The change in the sound velocity causes significant phase errors in the beamforming. These errors are further aggravated by the existence of near field inhomogeneities. The phase aberration can result in increase in side lobes of beam pattern, degradation in lateral and range resolution, and also range and lateral shifts [12].

Various phase aberration correction methods have been reported for the nonuniform aberrating layers with random reflector distributions [15]-[18]. Recently, two-dimensional phase aberration correction studies have been presented [19]-[21]. Also, there exist time delay estimation techniques [22] which can be also used in off-line correction of phase aberration in ultrasound imaging. However, realtime ultrasound imaging necessitates on-line correction of 


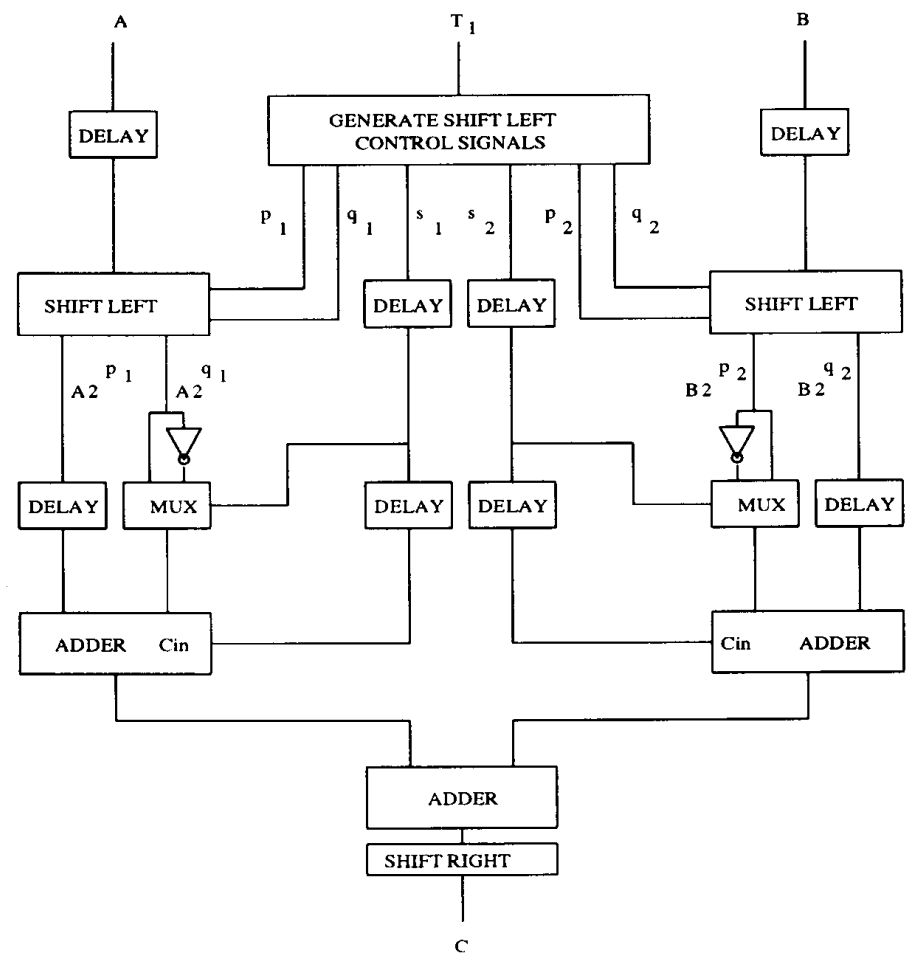

Fig. 3. Hardware structure of the interpolator.

phase aberrations. Hence, the computational efficiency of the correction algorithm is crucial in real-time applications.

The proposed technique is based on time delay estimation via the minimization of sum of absolute differences (SAD) between the sampled echo signals received at adjacent element pairs. For the sampled echo signals, the error measure is expressed as

$$
\epsilon(k)=\sum_{m=1}^{W}\left|s_{n-1}(m+k)-s_{n}(m)\right|
$$

where $W$ is the total number of samples, and $s_{n}(m)$ is the $m$ th sample of the echo signal received by $n$th element of the array. The time shift index minimizing this expression, $k$, is the sum of relative delays for focusing and aberration. Since the relative delays for focusing, $g$, are known, the aberration delay pattern can be obtained by a cumulative summation of the relative aberration delays:

$$
\Delta t_{n}=T_{s} \sum_{i=1}^{n} k_{i}-g_{i}
$$

where $T_{s}$ is the sampling period. To further increase the computational efficiency of the SAD technique the word length can be made shorter. The simulation results indicate that 1 bit word length is sufficient for phase aberration correction without a significant loss in the accuracy. Hence, the technique can be used on RF samples properly quantized to a single bit, where each addition reduces to a bit level exclusive-or (XOR).
Thus, the error expression becomes

$$
\epsilon(k)=\sum_{m=1}^{W} a_{m+k} X O R b_{m}
$$

where $a_{i}$ and $b_{i}$ are the one bit representation of echo samples $s_{n-1}(i)$ and $s_{n}(i)$, respectively, and the indexes used for element number are skipped. Obviously the hardware realization of this scheme is much simpler than that of the full word SAD. Reduction of the word length to a single bit can cause poor convergence in phase estimation from speckle noise. This effect can be avoided, however, by averaging the estimated phase patterns over a number of scan angles [18].

In order to compare the performances of the phase aberration correction techniques, the images of a standard graphitegel AIUM resolution phantom are reconstructed using the aberrated and non-aberrated (control) data sets. The data ${ }^{1}$ was acquired with a conventional phased-array transducer where the radio frequency (RF) A-scans were recorded from every possible combination of transmitter and receiver for all elements in a 64-element, 3.3-MHz array [15], [23]. Each Ascan was digitized after appropriate time gain compensation at a sampling rate of $17.76 \mathrm{MHz}$ with 10 -bit ADC. The aberrated data set was obtained using a distortion plate, made of RTV silicone rubber, which was placed between the transducer array and the phantom. The image reconstruction is carried out by digitally processing the recorded data to simulate the operation of a real-time digital imaging system. Since the

\footnotetext{
${ }^{1}$ Provided by Prof. Matthew O'Donnell of the University of Michigan.
} 


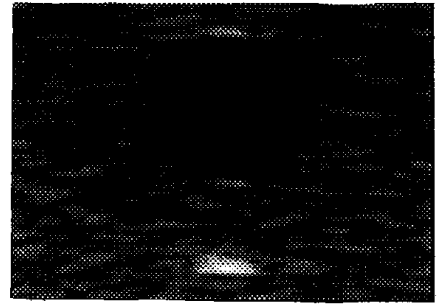

(a)

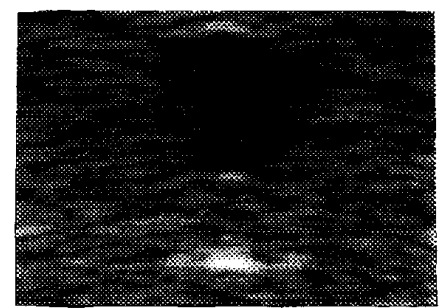

(d)

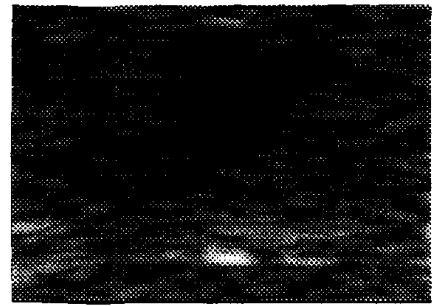

(b)

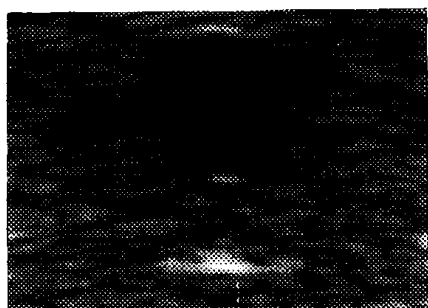

(e)

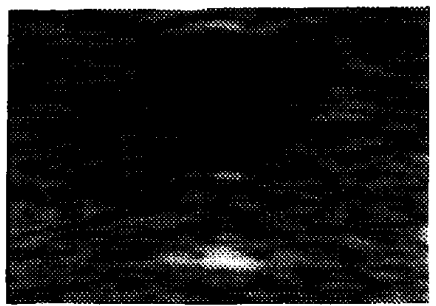

(c)

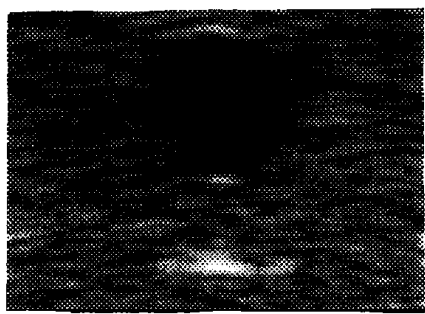

(f)

Fig. 4. Ultrasound images of a section of the phantom: (a) control, (b) aberrated, corrected using (c) cross-correlation, (d) SAD, (e) 1-bit SAD, and (f) 1-bit SAD averaged over 5 scan angles.

complete data set is recorded, it is possible to simulate any image reconstruction and beamforming technique.

Fig. 4 depicts the control, aberrated and the corrected images (a portion of the full sector image shown in Fig. 6). In reconstruction of these images, the sampling rate of the RF data sets are increased by 8 times using the linear interpolation technique discussed previously. Thus the phase aberration correction and beamforming operations are performed with about $142 \mathrm{MHz}$ sampling rate which corresponds to a phase accuracy of about $2 \pi / 43$. For each corrected image, the phase aberration pattern used in correction is estimated from the diffuse scatterers in three iterations on the central scan angle of the sector. The window length used for correlation and SAD is 4096 samples which corresponds to $20 \mathrm{~mm}$. It is observed from the images shown in Fig. 4 that the performance of the 1 -bit SAD is as good as the full word SAD and full word correlation. It is also noted that the averaging maintains the image quality performance of the 1-bit SAD while it eliminates the convergence problems.

Hardware realization of the phase aberration detection using 1-bit SAD method, requires delay elements and processing units for computation of the SAD terms for different shift indexes. The shift index of the minimum of the SAD terms corresponds to the time shift between the two channels. A processing unit consisting of three pipelined stages is developed (see Fig. 5) to form a flexible 1-bit SAD architecture by simply cascading a number of these units.

In the processing unit shown in Fig. 5, the binary data vectors in the SAD window, $\left(\left\{a_{m}\right\}_{m=1}^{W}\right.$ and $\left\{b_{m}\right\}_{m=1}^{W}$ in (5) are serially entered to the unit, from inputs $A$ and $B$. The unit also takes a binary reset input, $R$, and cumulative data inputs, SAD_MIN, SI_MIN, and SI. These are the minimum of SAD terms, the shift index corresponding to the minimum SAD, and the shift index indicating the shift count of the previous unit,

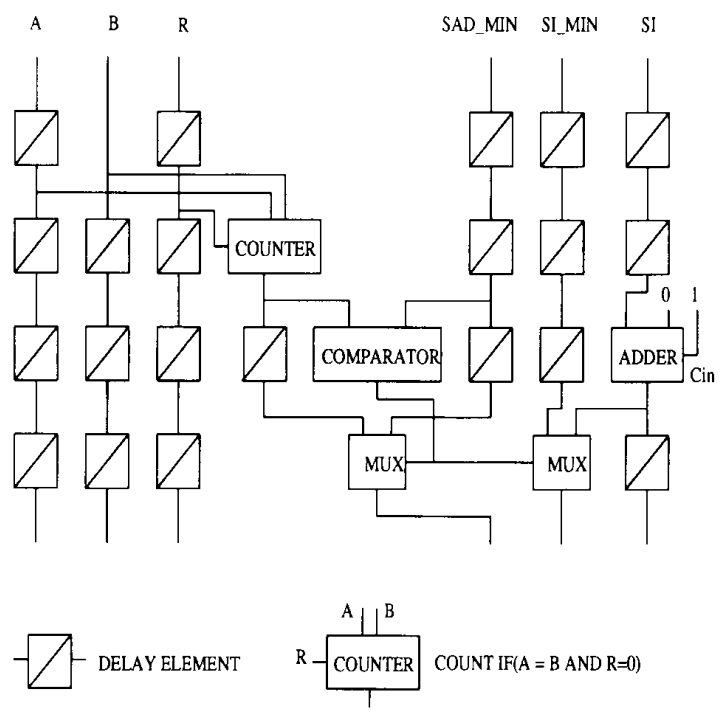

Fig. 5. Processing unit for the 1-bit SAD hardware.

respectively. The shift index is incremented and sent out to the succeeding unit. At any time, the shift index of the unit is regenerated. The computation of the SAD term corresponding to a different shift index is carried out using a counter which is updated by the result of XOR of the binary inputs. The counter is an upcounter which increments its value if the XOR of the input is 1 . When the last data inputs are processed, then the output of the counter becomes the result of the SAD of all binary data within the window. This is compared with the $S A D_{-} M I N$, and the smaller one and its corresponding index are sent out. 
The unit outlined above, computes the SAD term, $\epsilon(k)$, and then compares it with the input $S A D \_M I N=\min .\{\epsilon(k-1)$, $\epsilon(k-2), \ldots, \epsilon(0)\}$. It outputs the minimum of the SAD terms, $\{\epsilon(k), \epsilon(k-1), \ldots, \epsilon(0)\}$, and corresponding index. One can cascade $K$ stages of the unit to form a SAD network of $K$ shift indexes. Therefore, the minimum of $K$ SAD terms and associated indexes are computed in a pipelined manner and become available at the output of the last unit. The maximum number of the cascaded stages (i.e., the maximum value of shift index, $S I$, which equals to $K$ ), is determined by the size of the adder used for updating shift index, whereas the maximum number of input data (i.e., the length of the SAD window, $W$ ) is determined by the counter size.

\section{DigItal Beamforming}

In conventional phased-array ultrasound imaging, the radial data are converted to the raster format using digital scanconversion techniques. A typical digital scan converter maps the radial data to the nearest display pixel. This corresponds to the quantization of the radial coordinates to the nearest rectangular coordinates (see Fig. 1), where the data is forced into alignment with the display grid. Thus, it results in an annoying artifact called Moire pattern which is a well-defined pattern of holes in the image corresponding to the unaddressed pixels. Artifacts can be decreased by using two dimensional interpolation techniques [13], [6]. This significantly increases the computational cost of the scan conversion process.

The conversion process can be completely eliminated by performing the receive beamforming operations directly at the raster focal points of the imaging plane instead of the radial focal points. For this purpose, the timing data required for the receive beamforming must be computed for every raster focal point within the sector. Since the number of raster points in the sector is about 2.5 times the number of radial points, the number of receive beamforming operations increases by the same amount. This is a major reason why the analog systems employed beamforming at the radial focal points. However, it is possible to perform very fast beamforming operations using digital VLSI circuits. Here, we present a digital beamformer circuit and its VLSI implementation.

In order to make a comparison of the image quality performance of the receive beamforming at the radial and raster focal points, the sector images of the phantom are reconstructed using the two beamforming techniques. For this purpose the control (non-aberrated) RF data set, outlined in the previous section, is used. The sampling rate of the RF data is increased by 8 times using the linear interpolation scheme discussed previously, and the quadrature signal components are generated. The beamforming is performed using timing data computed for radial and raster receive focal points. The envelope detection is realized by taking the square root of the sum of the squares of in-phase and quadrature samples. The scan conversion which is employed in beamforming at the radial points, is realized using a first-order two-dimensional interpolation technique presented in [13]. Finally, a purely logarithmic compression is applied on the images to obtain a $60 \mathrm{~dB}$ dynamic range.
In the image reconstruction, the separation of the raster and radial focal points are chosen to be $\lambda_{0} / 2=0.23 \mathrm{~mm}$. The number of scan-slices $/ 90^{\circ}$-sector is 128 . The images consist of $512 \times 512$ pixels (Fig. 6). The transmit focal length is fixed at $80 \mathrm{~mm}$ away from the array, which corresponds to the position of the point reflector at center of the sector. It is observed from these images that the image obtained by beamforming at radial points is blurred due to the scan conversion. A detectable resolution improvement is achieved by performing the receive beamforming at the raster focal points.

In digital receive beamforming, the samples corresponding to a focal point are not synchronous. In order to find the signal value of a focal point, the samples must be suitably delayed and then added. This requires coherent addition of signals received by the array elements. However, a simple synchronization scheme designed for a regular data flow can not solve the synchronization problem in receive beamforming for sector imaging. This is due to the fact that the arrival time pattern of samples varies depending on the location of focal point within the sector.

In a straightforward approach, a "global" coherent summation scheme can be employed by using FIFO type registers at the adder front-end [4], [3], [24]: the samples from all channels are stacked in FIFO registers at each channel for synchronization, and then all of them are added. However, since the FIFO and adder sizes increase dramatically with the number array of elements, the scheme is not very feasible for implementation at the board level or in VLSI. Alternatively, the samples can be added recursively using partial sum registers [6]. But this technique is not particularly practical, because of the adder speed requirement.

An efficient receive beamforming hardware structure can be obtained by employing a "local" coherent addition technique where the total coherent summation of all samples corresponding to a focal point is obtained by a sequence of pairwise partial coherent summations [25]. For an $N$ channel system, at first, $N / 2$ partial coherent summations are obtained. Then, adjacent pairs of these partial sums are coherently added resulting in $N / 4$ new partial coherent sums. This procedure is repeated until the number of new partial coherent sums becomes unity, which is the total coherent summations of all samples. This approach results in an inverse binary tree like architecture for receive beamformer. For an $N$-element array, the network consists of $\log _{2}(N)$ stages, and the $k$ th stage has $N / 2^{k}\left(k=1,2, \cdots, \log _{2}(N)\right)$ processing units (see Fig. 7).

Each unit consists of FIFO registers and a full adder for coherent summation of its two input data. FIFO length for each stage is different; since there is no regularity in the arrival times of the signals corresponding to different focal points, the worst case FIFO lengths for the stages are determined by means of the computer simulation of the phased-array imaging system with a $90^{\circ}$ sector scanning.

The processing unit takes two data inputs, $A$ and $B$, along with two status bits, $S A$ and $S B$, and generates the coherent summation of the inputs, $D O$, with a corresponding output status bit, $S O$ (Fig. 8). Each status bit indicates that the data is valid. For a reliable real-time operation, the unit is designed in three pipelined stages: cross switch, FIFO, and 


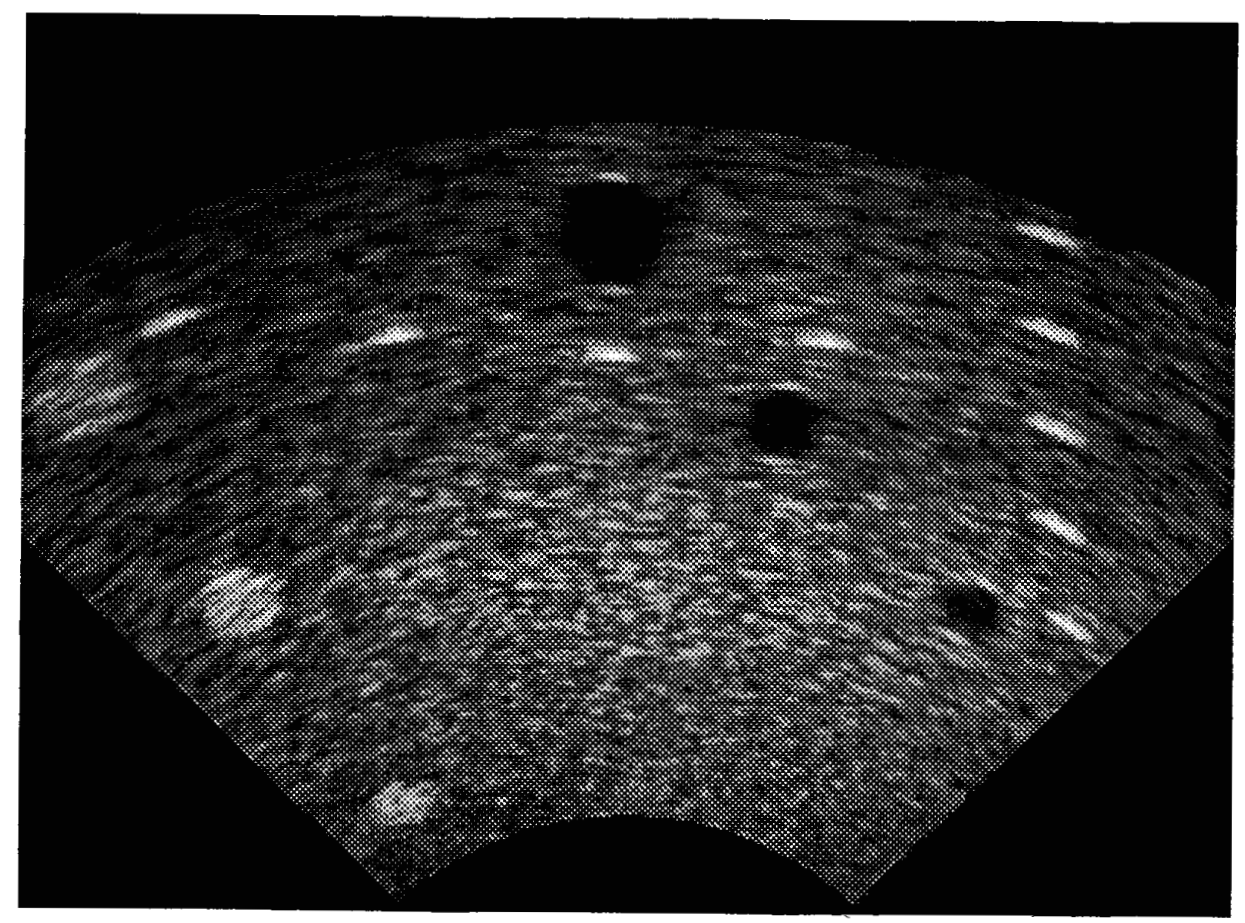

(a)

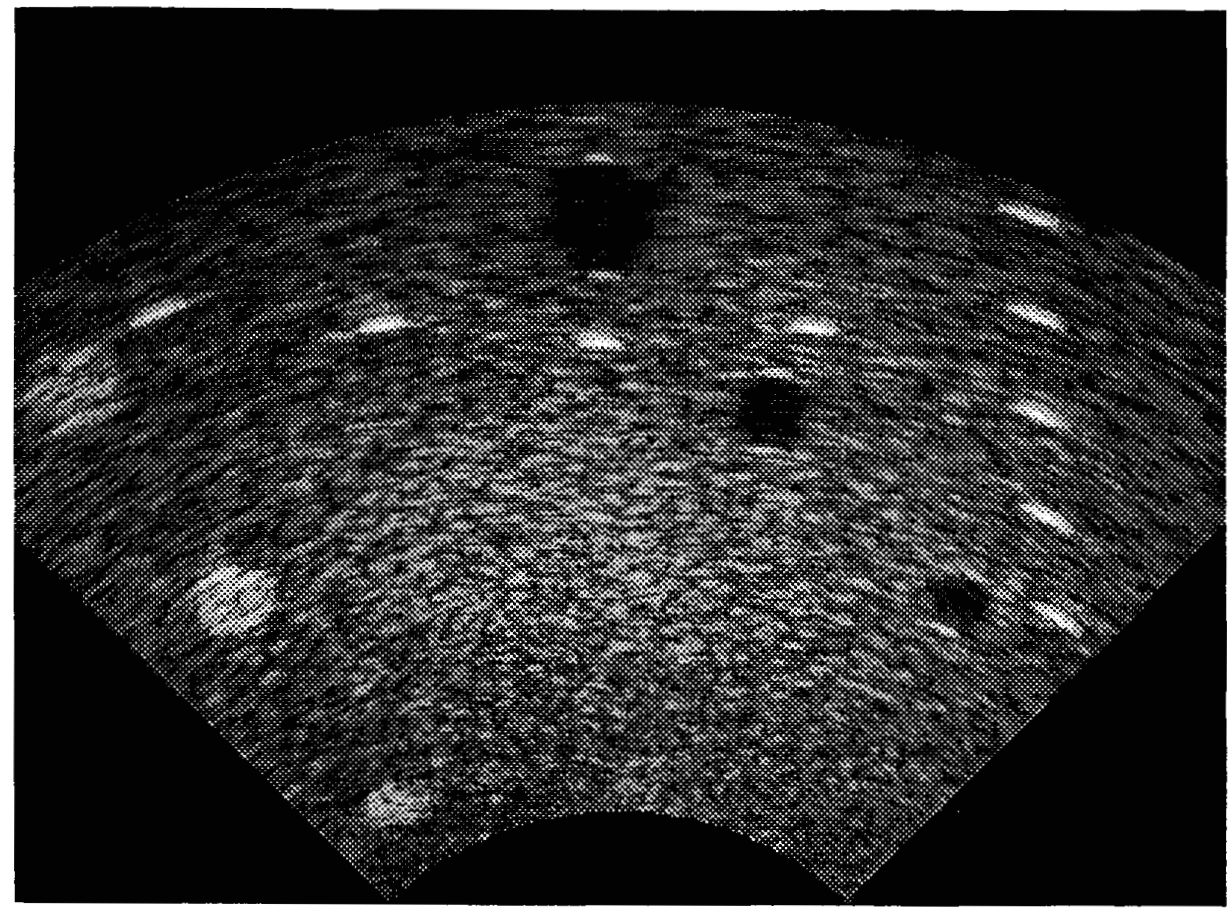

(b)

Fig. 6. Ultrasound sector images of the phantom reconstructed by receive beamforming at (a) radial and (b) raster focal points.

adder stages. The cross switch is a finite state machine which feeds the earlier of the inputs, $A$ or $B$, to the subsequent FIFO. After synchronization by FIFO, the data are fed to the full adder. The unit is reset by an external reset signal $(R)$ at the beginning of operation so that cross switch and FIFO pointers are set appropriately. Two non-overlapping clocks $\left(\phi_{1}\right.$ 


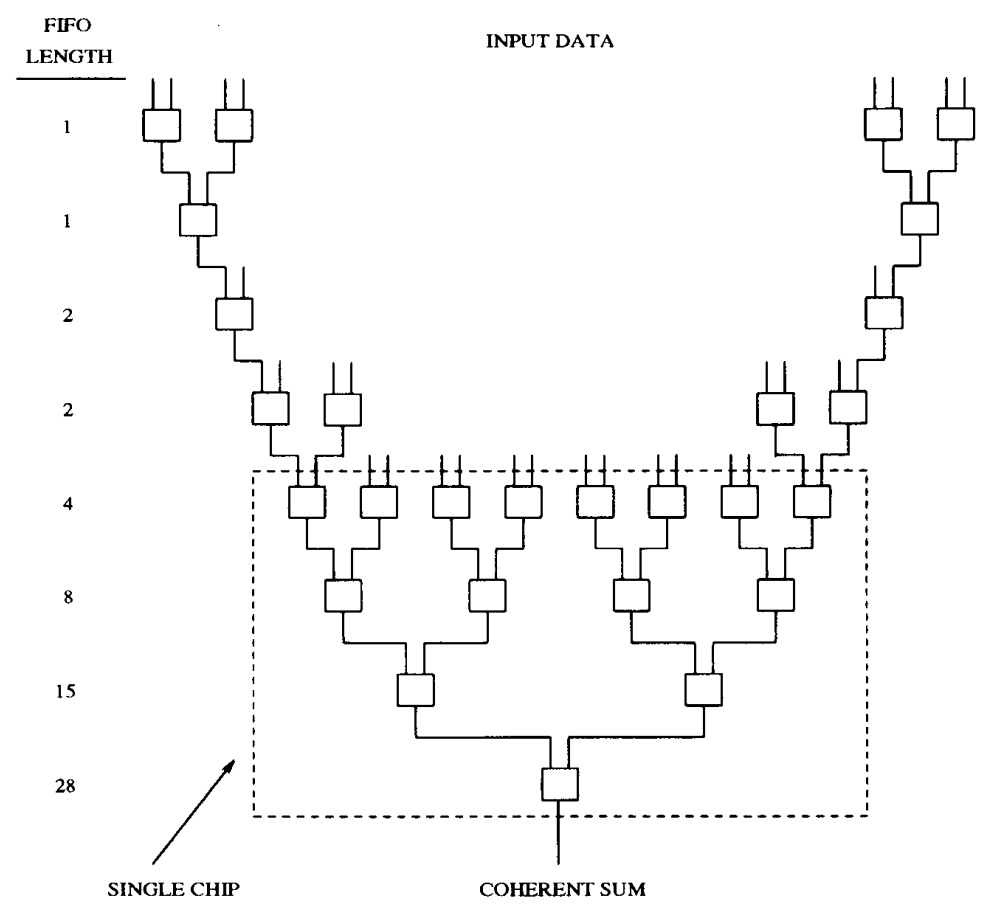

Fig. 7. Hardware structure of the beamformer.

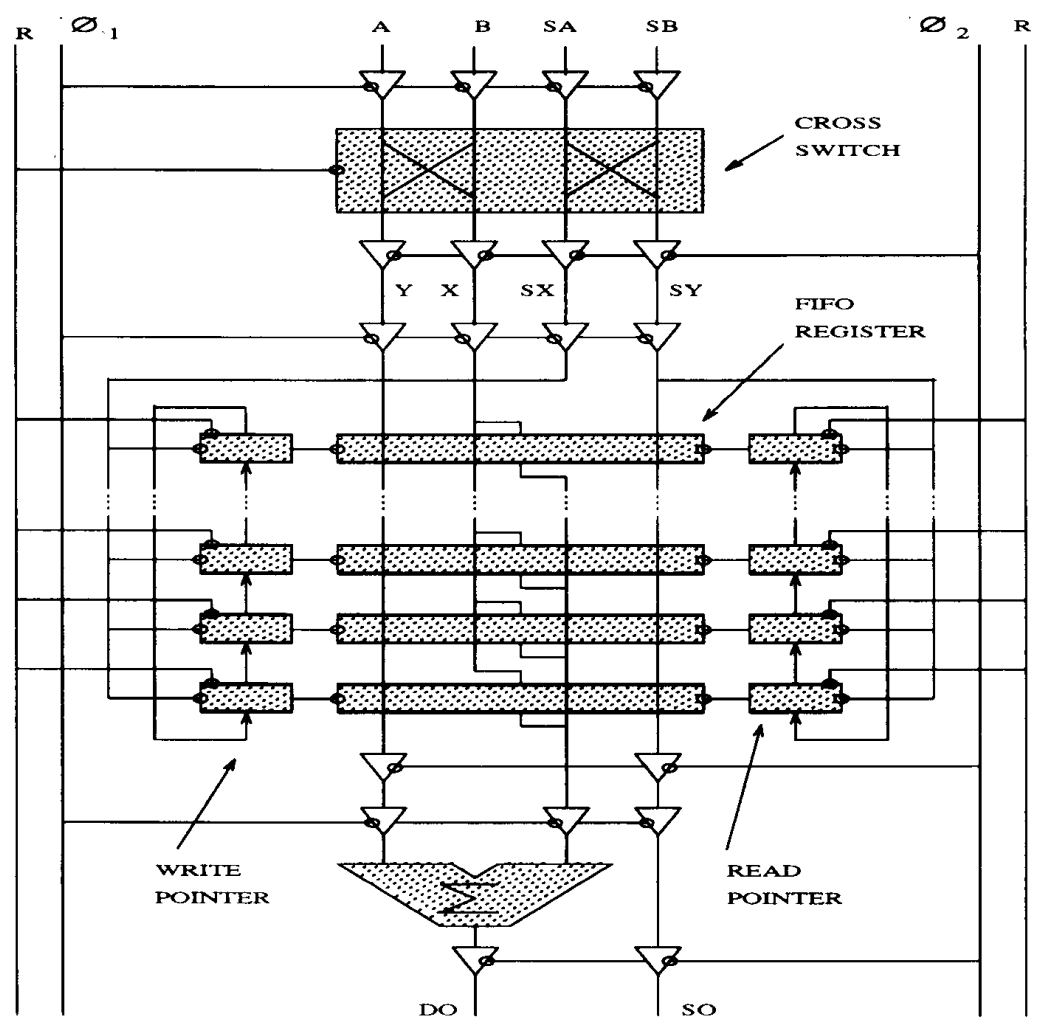

Fig. 8. Functional structure of one processing unit. 


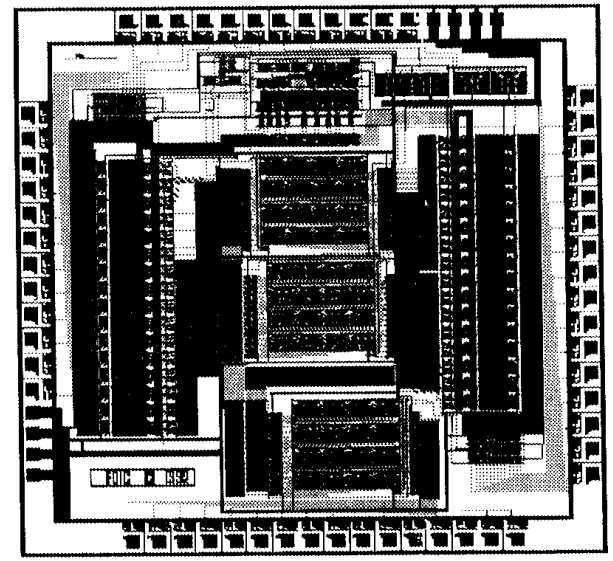

(a)

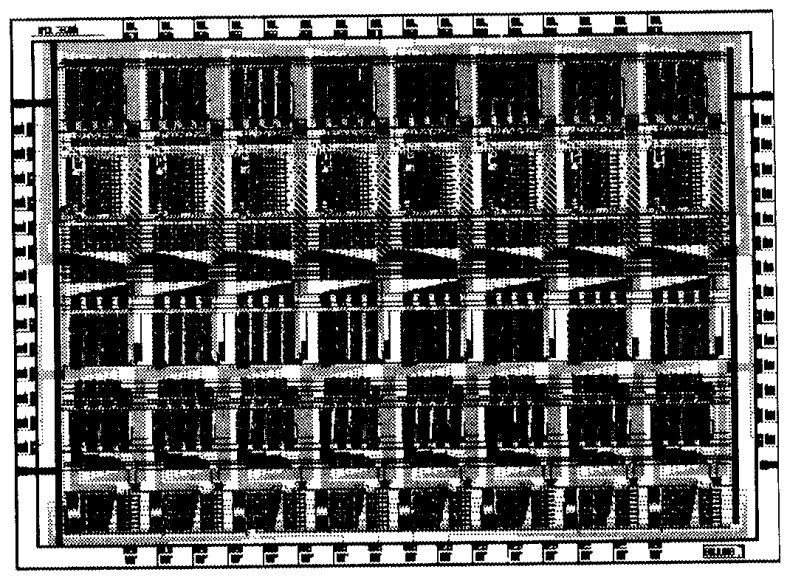

(b)

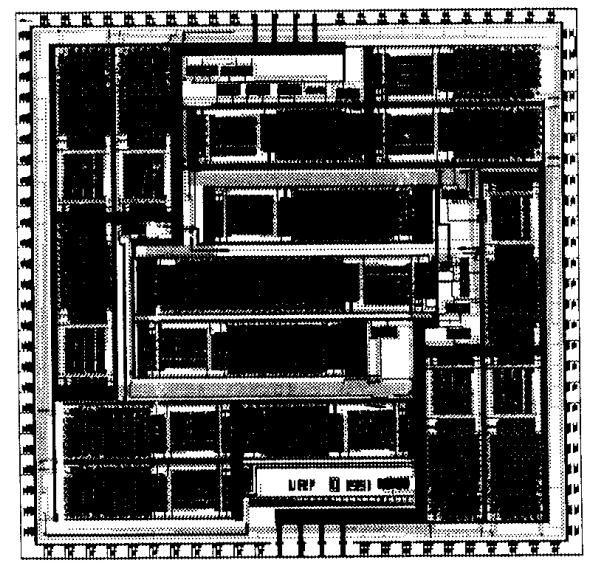

(c)

Fig. 9. Layouts of the designed chips: (a) Interpolator. (b) Phase estimator. (c) Beamformer.

and $\phi_{2}$ ) are used to control the pipelined operations and data flow.

\section{VLSI IMPLEMENTATION}

The proposed hardware structures are implemented in 1.5$\mu \mathrm{m} \mathrm{M}^{2} \mathrm{CMOS}$ technology using full-custom VLSI design techniques. In VLSI design of the chips, Magic, Hspice, and Irsim are used for layout editing, timing, and logic simulations, respectively. The layouts of the chips are shown in Fig. 9, and the characteristic parameters are outlined in Table I. The testing of the designed chips are easily accomplished by the functional test techniques, since the operations of the units are selectively probed by issuing proper test vectors [26].

The linear interpolator structure shown in Fig. 3 is implemented as a single chip. Each of the input samples and the interpolated output sample is 16 bits, whereas the $T_{1}$ input is 3 bits. The throughput of the chip is 40 Mega interpolations/s, which can meet the speed requirements of adaptive beamforming applications.

A phase aberration estimation chip, consisting of 8 cascaded units depicted in Fig. 5, is implemented. The word lengths are chosen sufficiently long to offer the chip to be used for sufficiently large window sizes and to be cascaded for larger shift indexes. The single chip can be used for window sizes up to 4096 samples with 8 shift indexes, whereas for shift indexes up to 128 , the number of chips to be cascaded is the smallest greater integer of $K / 8$. A single chip can be used in phase aberration detection, where the shift index of adjacent array element pairs does not exceed 8 for sampling rates up to $50 f_{o}$, and for phase aberration variation within a cycle. On the other hand, the window size is sufficiently long for phase aberration correction from both point reflectors and the diffuse scatterers for sampling rates up to $50 f_{o}$. As an example, the SAD window of size 4096 corresponds about $20 \mathrm{~mm}$ for $f_{o}=3.33 \mathrm{MHz}$ and sampling rate of $43 f_{o}$, which are the parameters used in 


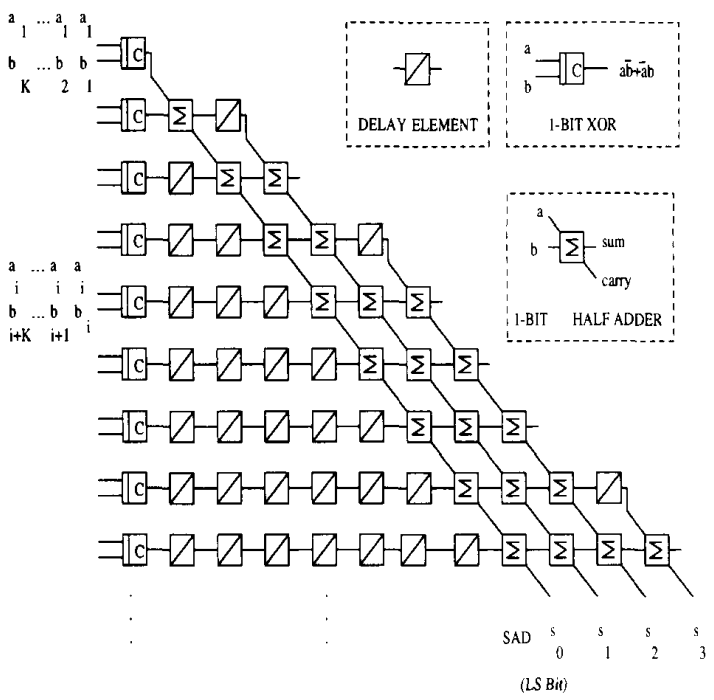

Fig. 10. High throughput architecture for 1-bit SAD.

the phase aberration correction of the images shown in Fig. 4.

The computation at each pipelined stage is completed in about 20 nsec resulting in a pipelined speed of 50 Mega operations/s. Thus, the throughput of the chip is about $50 / \mathrm{W}$ Mega outputs/s. This enables the phase aberration detection of about $12 \mathrm{~K} / \mathrm{s}$ with $W=4096$. In sector imaging, the phase aberration correction can be applied at each frame where the phase correction iterations can be carried out on the consecutive frames. Also, the angle dependent correction can be carried out for a number of scan angle groups on each frame. For 128 scan angles/frame and 30 frames/s, the number of the phase aberration correction operations is 3840 . Hence, the speed of the designed chip is adequate for phase aberration correction at each scan angle for every frame.

A receive beamformer for $N=16$ is realized by connecting 15 processing units in an inverse binary tree structure to form the core of the chip. The chip consists of the lowest four stages of the network for $N=256$, outlined by a dashed rectangle in Fig. 7. Since the lowest four stages have the longest FIFO's, one can connect the chips to form a network for $N \leq 256$. Maximum throughput of the chip is 40 Mega beamforming operations/s. The chip has 16 multiplexed inputs and 1 multiplexed output, 16 bits plus 1 status bit each. Overall result of coherent addition is truncated to 16 bits from 20 bits, while the maximum dynamic range requirement is about 12 bits.

\section{DISCUSSION AND CONCLUSION}

In this paper, we present cost-effective digital beamforming techniques with high timing accuracy, phase aberration correction, and efficient receive focusing. Performances of these techniques are tested and illustrated using the phased-array experimental RF data sets. Special purpose VLSI circuits based on these techniques are proposed. Single-chip realizations of
TABLE I

Characteristic Parameters of the Designed ChIPS

\begin{tabular}{lccc}
\hline Parameter & Interpolator & Phase Estimator & Beamformer \\
\hline Die size $\left(\mathrm{mm}^{2}\right)$ & $2.8 \times 3.0$ & $4.4 \times 6.0$ & $6.6 \times 6.7$ \\
Transistor count & 5 & 28 & 40 \\
(K) & 64 & 64 & 100 \\
Pin count & 40 & 50 & 40 \\
Max. clock freq. & 40 & $50 / \mathrm{w}$ & 40 \\
(MHz) & & & \\
Max. throughput & $($ Mega outputs/s) & &
\end{tabular}

the circuits using full-custom CMOS VLSI design techniques are outlined.

In the digital front-end hardware as depicted in Fig. 2, the processing units can be realized using the designed chips (interpolator, phase estimator, and beamformer). Both of the re-sampling and the beamforming operations can be handled by the beamformer chip(s), where the input status entry controls the sample selection. For an $N$-channel phased-array imaging system, the number of chips for upsampling, phase estimation, and beamforming are $N, N-1$, and smallest greater integer of $N / 16$, respectively.

The proposed front-end circuits involve sampling of RF echo signals received from all array channels. This is a major limitation in all digital beamforming schemes as well as the one discussed in this work. The speed of the ADC's, however, can be at the Nyquist rate, since a special interpolation circuit is also employed to decrease the effects of delay quantization.

The phase estimator chip can meet the speed requirements of one- and two-dimensional angle-dependent phase aberration correction applications. Should a faster phase-error estimation be required, such as for correction of both angle- and rangedependent phase errors, the proposed 1-bit SAD hardware structure can be modified to increase the throughput further. This can be achieved in a systolic scheme by employing bit level pipelining and parallelism [27]. Such an architecture with very high throughput is depicted in Fig. 10. In this architecture, the binary data, $\left\{a_{m}\right\}_{m=1}^{W}$ and $\left\{b_{m+k}\right\}_{m=1}^{W}$ are applied to the inputs in parallel. There are three bit-level processing elements: delay, half adder, and XOR elements. Since all operations are pipelined at bit-level, the computation time of a SAD term is determined by a bitwise XOR operation. All of the SAD terms, $\{\epsilon(i)\}_{i=1}^{K}$, are computed at $K$ pipelined clocks. The minimum of the SAD terms can be found using a fast comparator or a sorter. VLSI design complexity of the architecture given in Fig. 10 is very low. Since the area of the architecture increases with $W^{2} / 2$, it is suitable for the realizations with a reasonably small $W$.

Proposed receive beamforming scheme is an efficient structure that possesses the following advantages: 1) small storage for data synchronization, 2) flexibility in applications with different number of array elements, 3 ) operating capability at real-time rate, and 4) feasibility for custom chip(s) implementation. VLSI implementation for large $N$ is not very feasible because of the large chip area and large number of I/O's. The modularity of the structure can be employed effectively, however, by proper choice of FIFO sizes, and by using the 
beamformer chips with $N=16$. Furthermore, the designed beamformer can perform real-time receive beamforming at the radial or raster focal points.

\section{ACKNOWLEDGMENT}

The authors would like to thank to Prof. Dr. Matthew O'Donnell of University of Michigan, for kindly providing the ultrasound phase array RF data sets of the phantom, and for his valuable helps in digital processing of the data. The authors would like to acknowledge the cooperations of C. Aydın, A. E. Kolagasıoglu, M. Ş.Toygar, I. A. Baktır, R. Tahboub, E. Erzin, F. Kılıç, and M. H. Asyalı, in VLSI implementation of the beamformer chip.

\section{REFERENCES}

[1] J. F. Havlice and J. C. Taenzer, "Medical ultrasound imaging: An overview of principles and instrumentation," in Proc. IEEE, vol. 67 pp. 620-641, April 1979.

[2] M. E. Schafer and P. A. Lewin, "The influence of front-end hardware on digital ultrasonic imaging," IEEE Trans. Sonics. Ultrasan., vol. 31 , pp. 295-306, July 1984

[3] M. O'Donnell, "Applications of VLSI circuits to medical imaging," in Proc. IEEE, vol. 76, pp. 1106-1114, Sept. 1988

[4] J. P. Stonestorm and W. A. Anderson, "Custom NMOS chip for medica ultrasound," VLSI Design, pp. 44-49, May 1982.

[5] M. O'Donnell et al., "Real-time phased-array imaging using digital beam forming and autonomous channel control," in Proc. 1990 IEEE Ultrason Symp., pp. 1499-1502, 1990.

[6] R. M. Lutolf, A. Vieli, and S. Basler, "Ultrasonic phased-array scanner with digital echo synthesis for Doppler echocardiography," IEEE Trans. Ultrason. Ferroelec. Freq. Contr., vol. 36, pp. 494-506, Sept. 1989.

[7] R. A. Harris et al., "Ultimate limits in ultrasonic image resolution," Ultrasound in Med. and Biol., vol. 17, pp. 547-558, 1991.

[8] M. J. Foster and H. T. Kung, "The design of special purpose VLSI chips," IEEE Computer, pp. 26-40, Jan. 1980.

[9] D. K. Peterson and G. S. Kino, "Real-time digital image reconstruction A description of imaging hardware and an analysis of quantization errors," IEEE Sonics. Ultrason., vol. 31, pp. 337-351, July 1984.

[10] R. G. Pridham and R. A. Mucci, "Digital interpolation beamforming for low-pass and bandpass signals," in Proc. IEEE, vol. 67, pp. 904-919, April 1979.

[11] Y. C. Lim and S. R. Parker, "FIR filter designed over a discrete powers-of-two coefficient space," IEEE Trans. Acoust., Speech, Signal Processing," vol. 31, pp. 583-590, June 1983.
[12] G. E. Trahey, P. D. Freiburger, L. F. Nock, and D. C. Sullivan, "In vivo measurements of ultrasonic beam distortion in the breast," Ultrasonic Imaging, vol. 13, pp. 71-90, 1991

[13] S. Leavitt and B. F. Hunt and H. G. Larsen, "A scan conversion algorithm for displaying ultrasound images," Hewlett-Packard $J$, vol. 34, pp. 30-34, Oct. 1983.

[14] O. T. von Ramm and S. W. Smith, "Beam steering with linear arrays," IEEE Trans. Biomed. Eng., vol. 30, pp. 438-452, Aug. 1983.

[15] S. W. Flax and M. O'Donnell, "Phase-aberration correction using signals from point reflectors and diffuse scatterers: Basic principles," IEEE Trans. Ultrason. Ferroelec. Freq. Contr., vol. 35, pp. 758-767, Nov. 1988.

[16] D. Zhao and G. E. Trahey, "Comparisons of image quality factors for phase aberration correction with diffuse and point targets," IEEE Trans. Ultrason. Ferroelec. Freq. Contr., vol. 38, pp. 125-132, Mar. 1991.

[17] F. Wu, et al., "Optimal focusing through aberrating media: A comparison between time reversal mirror and time delay correction techniques," in Proc. 1991 IEEE Ultrason. Symp., pp. 1195-1199, 1991.

[18] M. Karaman, A. Atalar, and H. Köymen, "Adaptive digital beamforming for real-time phased array ultrasound imaging," in Proc. 1991 IEEE Ultrason. Symp., pp. 1207-1210, 1991

[19] G. E. Trahey and P. D. Freiburger, "An evaluation of transducer design and algorithm performance for two dimensional phase aberration correction," in Proc. 1991 IEEE Ultrason. Symp., pp. 1181-1187, 1991

[20] M. O'Donnell and P. C. Li, "Aberration correction on a two-dimensional anisotropic phased array," in Proc. 1991 IEEE Ultrason. Symp., pp. 1189-1193, 1991

[21] R. Kanda, Y. Sumino, K. Takamizawa, and H. Sasaki, "An investigation of wavefront distortion correction: Correction using averaged phase information and the effect of correction one and two dimensions," in Proc. 199I IEEE Ultrason. Symp., pp. 1201-1206, 1991.

[22] K. Scarbrough, N. Ahmed and G. C. Carter, "On the simulation of a class of time delay estimation algorithms," IEEE Trans. Acoust. Speech, Signal Process., vol. 29, pp. 534-539, June 1981.

[23] M. O'Donnell and S. W. Flax "Phase-aberration correction using signals from point reflectors and diffuse scatterers: Experimental results," IEEE Trans. Ultrason. Ferroelec. Freq. Contr., vol. 35, pp. 768-774, Nov 1988

[24] T. H. Song and S. B. Park, "A new digital phased array system for dynamic focusing and steering with reduced sampling rate," Ultrasonic Imaging, vol. 12, pp. 1-16, 1990.

[25] M. Karaman, E. Kolăgasıŏlu, and A. Atalar, "A VLSI receive beamformer for digital ultrasound imaging," in Proc. ICASSP'92, pp. V-657$660,1992$.

[26] J. A. Abraham and W. K. Fuchs, "Fault and error models for VLSI," in Proc. IEEE, vol. 74, pp. 639-654, May 1986

[27] H. T. Kung, "Why systolic architectures?" IEEE Computer, pp. 37-46, Jan. 1982. 